\section{Immune-mediated polyarthritis in fourteen cats (2009-2018)}

\section{Florence Wootton ${ }^{1}$, Sorrel Langley-Hobbs ${ }^{1}$, Fiona Whitworth ${ }^{1}$, Anna Threlfall' Thomas Anderson ${ }^{3}$, Craig Breheny4, Vicki Black ${ }^{1}$}

1 Bristol Vet School, Bristol, United Kingdom

2 Davies Veterinary Specialists, Higham Gobion, United Kingdom

3 Dick White Referrals, Six Mile Bottom, United Kingdom

4 Edinburgh University, Edinburgh, United Kingdom

\section{OBJECTIVES}

To describe the clinical features and outcome in cats with non-erosive immune-mediated polyarthritis.

\section{METHODS}

Multicentre retrospective case series. Cats with nonerosive immune-mediated polyarthritis were identified by searching five hospital databases between 2009 and 2018. Data including signalment, history, investigation findings including presence of a trigger and category (infectious, gastrointestinal, or neoplastic), and treatment was analysed. Referring vets were contacted for follow-up.

\section{RESULTS}

Fourteen cases were identified; there was no obvious sex predisposition (7 MN, $2 \mathrm{ME}, 5 \mathrm{FN}$ ), median age was 8.5 years (range 1-14 years), and most common breeds were domestic shorthairs (7) and Maine coons (3). Common clinical signs included lameness (14/14) and pyrexia (6/12). In half of the cases (7/14) a trigger was identified; this was considered to be infectious in four cats (FIV 2/4, infectious discospondylitis $1 / 4$, pneumonia 1/4) and gastrointestinal in three cats.

Ten cats received immunosuppressive therapy (prednisolone $8 / 10$, ciclosporin $1 / 10$, prednisolone and chlorambucil $1 / 10$ ). In four cats adjunctive immunosuppressants were later instituted (chlorambucil 3/4, ciclosporin 1/4) due to poor response (2/4) or side-effects (2/4). The four cats considered to have an infectious trigger received anti-inflammatories $+/-$ antimicrobials. At short-term follow-up four cases were euthanased due to poor response (3/4) or drug side-effects (1/4).

\section{STATEMENT (CONCLUSIONS)}

Pyrexia is not a consistent feature in cats with immunemediated polyarthritis and a trigger, specifically infectious or gastrointestinal disease should be considered. Prognosis appears fair, some cats experienced drug side-effects or had a poor response to immunosuppressive monotherapy.

\section{Does duration of clinical signs prior to diagnosis affect outcome in dogs with immune-mediated polyarthritis}

\section{Simon Mcmanus, Lucy Barker, Sophie Adamantos, Vicki Black}

University of Bristol, Bristol, United Kingdom

\section{OBJECTIVES}

To explore outcome in dogs diagnosed with primary non-erosive immune-mediated polyarthritis and to evaluate whether duration of clinical signs for more than 30 days prior to diagnosis affects risk of relapse.

\section{METHODS}

Retrospective single referral-centre study. The hospital database was searched January 2009 to August 2018 for dogs with a diagnosis of idiopathic non-erosive IMPA. Data extracted included signalment, duration of clinical signs, outcome and relapse episodes. Long-term followup was achieved by contacting referring veterinary practices. Data was recorded descriptively and categorised. A Chi squared test was used to assess whether clinical signs of $>30$ days duration prior to diagnosis was associated with a risk of relapse.

\section{RESULTS}

One hundred and one dogs were identified. Long-term follow-up was available for 75 dogs. Median age at diagnosis was 4.49 years (range $0.38-11.02$ years). Median duration of clinical signs was 30 (range 1-632) days, $37 / 75$ cases $(49 \%)$ relapsed, with a median relapse time of 119 (range 3-1410) days after diagnosis. There was no association between duration of clinical signs and risk of relapses $(p=0.54)$. Fifty-six dogs were alive at the time of follow-up, $12 / 19(63 \%)$ died or were euthanised as a result of their disease.

\section{STATEMENT (CONCLUSIONS)}

Long-term prognosis in this cohort of dogs with primary non-erosive immune-mediated polyarthritis was fair, with a high risk of relapse $(49 \%)$ and death due to disease (16\%). Although duration of signs prior to diagnosis was highly variable, this was not associated with risk of relapse. 